\title{
Eosinophilic myocarditis associated with dense deposits of eosinophil cationic protein (ECP) in endomyocardium with high serum ECP
}

\author{
M Arima, T Kanoh
}

\begin{abstract}
A case of eosinophilic myocarditis following high serum levels of eosinophil cationic protein (ECP) is described. A 27 year old woman was admitted with New York Heart Association (NYHA) class III congestive heart failure. A haematological study showed hypereosinophilia with degranulation and vacuoles; the total eosinophil count was $7980 / \mathrm{ml}$ and the ECP serum concentration was noticeably high at $150 \mathrm{ng} / \mathrm{ml}$. Endomyocardial biopsy from the right ventricle showed infiltration of eosinophils and dense deposits of ECP in the endocardium as well as the myocardium. Steroid treatment returned the total eosinophil count and serum ECP to normal, with satisfactory improvement in clinical features. Eosinophilia may cause cardiac damage, and this report confirms that eosinophil degranulation is toxic. Thus, serum ECP seems to be a reliable indicator for diagnosis and for determining treatment parameters of eosinophilic myocarditis.

(Heart 1999;81:669-675)
\end{abstract}

Keywords: eosinophilic myocarditis; eosinophilia; eosinophil cationic protein; endomyocardial biopsy

Endomyocardial disease has been linked to many diseases that cause eosinophilia. Eosinophilic myocarditis, Loffler's endocarditis, Davis disease, and endomyocardial fibrosis are different stages of a single disease entity caused by eosinophil mediated cardiac injury. ${ }^{1}$ Eosinophil degranulation produces several toxic proteins, which may play an important role in the pathogenesis of this cardiac disorder. We report a case of eosinophilic myocarditis in which the myocardium showed a noticeable infiltration of eosinophils and dense deposits of eosinophil cationic protein (ECP) as well as high serum ECP.

\section{Case report}

A 27 year old woman was admitted with NYHA class III congestive heart failure, oedema of the lower extremities, and slight jugular vein enlargement. She had no history of drug allergy nor a family history of heart disease. Dyspnoea on exertion and oedema had developed in the two weeks before admission. On physical examination, her blood pressure was $120 / 84 \mathrm{~mm} \mathrm{Hg}$, with a regular pulse rate of 90 beats $/ \mathrm{min}$. Although there was no audible cardiac murmur, friction rub was heard in the apex. Fine bubbling rales were heard at the bilateral bases. An ECG showed a normal sinus rhythm with low voltage in limb leads and terminal $\mathrm{T}$ wave inversion in leads I, II, III, aVL, aVF, and V2-V6. Chest radiography showed a cardiothoracic ratio of $54 \%$ with congestive lung and bilateral pleural effusion, and cross sectional echocardiography revealed moderate pericardial effusion and a diffusely thickened left ventricular wall (interventricular septum $13 \mathrm{~mm}$ and posterior wall $12 \mathrm{~mm}$ ), with a slight global reduction in systolic performance (ejection fraction 59\%). The results of a routine haematological study showed a leukocyte count of $15200 / \mathrm{ml}$, and $19.7 \%$ neutrophils, $25.8 \%$ lymphocytes, $2 \%$ monocytes, and $52.5 \%$ eosinophils. The total eosinophil count was $7980 / \mathrm{ml}$. We observed that the eosinophils were degranulated and that vacuoles had formed. Serum ECP, which is one of several toxic proteins derived from eosinophil degranulation, and which may play an important role in the pathogenesis of the cardiac disorder, was $150 \mathrm{ng} / \mathrm{ml}$ (normal, $<15 \mathrm{ng} / \mathrm{ml}$ ). We observed a slight rise in both serum creatine phosphokinase (122 IU/1 (normal 9-93)) and lactic dehydrogenase (485 IU/1 (normal 180460)). However, levels of aspartate aminotransferase (22 IU/1), alanine aminotransferase (20 $\mathrm{IU} / \mathrm{l}), \mathrm{C}$ reactive protein $(0.003 \mathrm{~g} / \mathrm{l})$, and serum $\mathrm{IgE}$ concentration (317 IU/ml (normal 18501)) were within normal limits. Stool examinations for ova and parasites were negative, as were serological tests for antibodies to viruses. Finally, the results of a bone marrow examination were normal except for a pronounced increase in the number of eosinophils.

The patient became asymptomatic 10 days after treatment with oxygen and diuretics. There was a satisfactory improvement in cardiomegaly, but the total eosinophil count and serum ECP had not dropped and, as before, cross sectional echocardiography showed mild pericardial effusion and a diffusely thickened left ventricular wall. Cardiac catheterisation
Dr Arima

Accepted 2 November 1998 

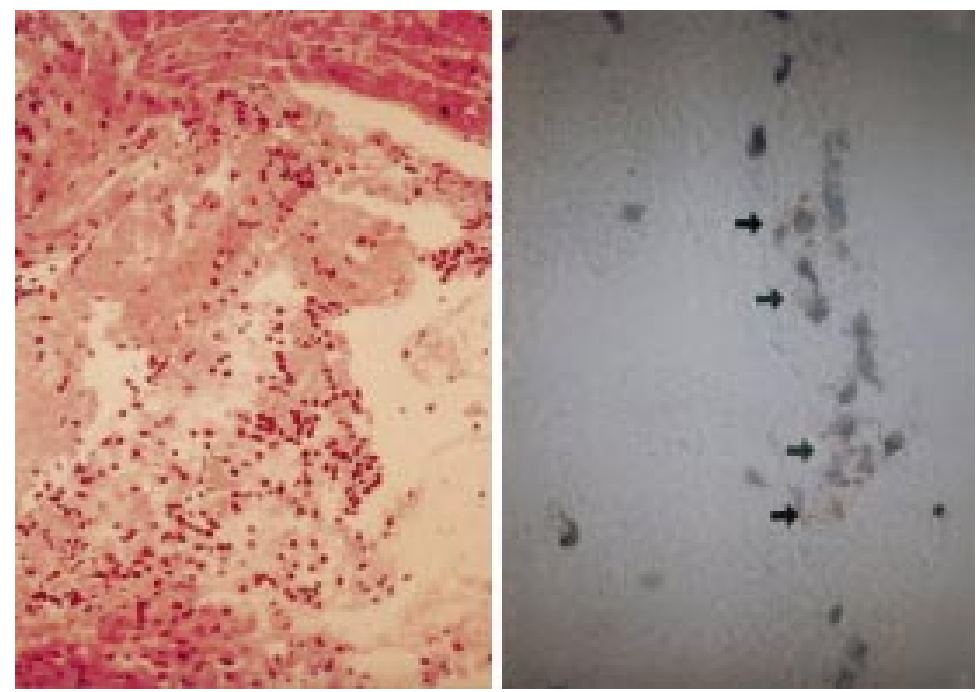

Figure 1 Endomyocardial biopsy (left) from the right ventricle showing slight necrosis and degeneration of myocytes, interstitial fibrosis, and infiltration of lymphocytes admixed with marked eosinophils (haematoxylin and eosin stain, original magnification $\times 40$ ).

Immunostaining with EG2 (arrow) (right), a monoclonal antibody directed against the secreted form of ECP, showed a great number of activated eosinophils (original magnification $\times 100$ ). reaction to antibiotics, ${ }^{3}$ may all result in hypereosinophilia and, thus, eosinophilic endomyocardial disease. However, in most patients with hypereosinophilia and eosinophilic endomyocardial disease, the cause of the disease is unknown. Eosinophils can damage tissues - for example, heart cells, and the resulting cardiac damage may be related to the toxic effects of activated eosinophils. Some studies have implicated eosinophil granule proteins in the progression of endomyocardial lesions by showing deposits of a major basic protein and ECP in the area of cardiac injury. ${ }^{45}$ Endomyocardial biopsy is helpful in making a correct diagnosis, but it cannot be performed in all patients suspected of having endomyocardial disease as it is too invasive. In our patient, there was a close correlation between the number of degranulated eosinophils in her circulation and ECP serum concentration. This shows that serum ECP can be a valuable and objective parameter in monitoring disease activity, particularly after treatment. ${ }^{6}$

When an endomyocardial biopsy identifies early stage eosinophilic myocarditis, the disease can be treated with corticosteroids which might prevent cardiac damage. ${ }^{7}$ Thus, it is important to make the diagnosis and carry out treatment as soon as possible to prevent the condition worsening. Endomyocardial biopsy cannot be performed repeatedly, but measuring serum ECP is a reliable and accurate method of gauging the effectiveness of any treatment. As changes in ECP serum concentration correspond with the degranulated eosinophil count, serum ECP may reflect cardiac damage. Therefore, it is useful to measure serum ECP in patients with eosinophilia following diagnosis and during treatment.

In our patient, eosinophil infiltration and dense deposits of ECP were seen in the endocardium and the myocardium. After steroid treatment, which reduced the total eosinophil count and the serum ECP, the diffusely thickened left ventricular wall and pericardial effusion disappeared and cardiac function returned almost to normal. Although pericardial paracentesis was not performed, this suggests that eosinophil mediated cardiac injury extended to the endomyocardium and the pericardium, indicating panmyocarditis. To our knowledge, this is the first reported case in which dense deposits of ECP have been detected in the endomyocardium through monitoring ECP serum concentrations. Although the mechanisms responsible for the activation and release of ECP after eosinophil degranulation were not identified, eosinophil activation markers were found in both the blood and cardiac tissue. Thus, eosinophilia may cause cardiac damage, and our findings confirm the presence of toxicity resulting from eosinophil degranulation. Therefore, ECP serum concentrations seem to be a valuable and foolproof indicator for diagnosis and for determining treatment parameters of eosinophilic myocarditis. 
1 Olsen EGJ, Spry CJF. Relation between eosinophilia and Olsen EGJ, Spry CJF. Relation between eosinophilia and 54

2 Tai PC, Spry CJF, Peterson C, et al. Monoclonal antibodies distinguish between storage and secreted forms of eosinophil cationic protein. Nature 1984;309:182-4

3 Markus CK, Chow LH, Wycoff DM, et al. Pet food-derived penicillin residue as a potential cause of hypersensitivity myocarditis and sudden death. Am f Cardiol 1989;63 1154-6.

4 Tai PC, Ackerman SJ, Spry CJF, et al. Deposits of eosinophil granule proteins in cardiac tissues of patients with eosinophilic endomyocardial disease. Lancet 1987;i:643-7. De Mello DE, Liapis H, Jureidini S, et al. Cardiac localization of eosinophil-granule major basic protein in acute necrotizing myocarditis. N Engl f Med 1990;323:1542-5.
Desreumaux P, Janin A, Dubucquoi S, et al. Synthesis of Desreumaux P, Janin A, Dubucquoi S, et al. Synthesis of
interleukin-5 by activated eosinophils in patients with eosiinterleukin-5 by activated eosinophils in patients
nophilic heart diseases. Blood 1993;82:1553-60.

$7 \mathrm{Kim}$ CH, Vlietstra RE, Edwards WD, et al. Steroidresponsive eosinophilic myocarditis: diagnosis by endomyocardial biopsy. Am f Cardiol 1984;53:1472-3.

\section{IMAGES IN CARDIOLOGY}

\section{Aortic coarctation diagnosed by magnetic resonance angiography}

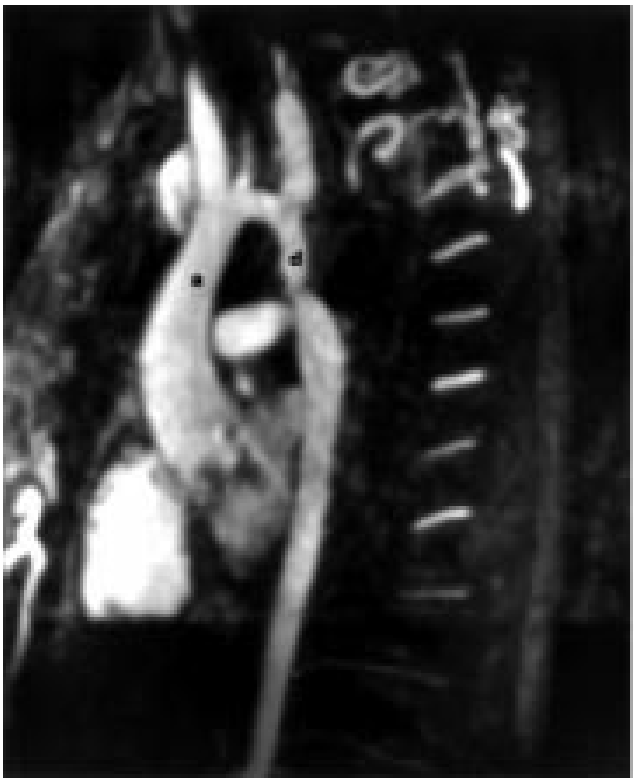

A 20 year old man was found to be hypertensive on routine screening. Examination revealed an absent right and barely palpable left femoral arterial pulse. Radiofemoral delay was present and pulsations were palpable over both scapulae. Parasaggital gadolinium enhanced magnetic resonance angiography (left) demonstrates a uniform $3 \mathrm{~cm}$ narrowing in the proximal descending aorta $(d)$, which terminates in a discrete stricture before returning to a normal calibre (a, ascending aorta). Internal mammary artery hypertrophy is noted in the retrosternal

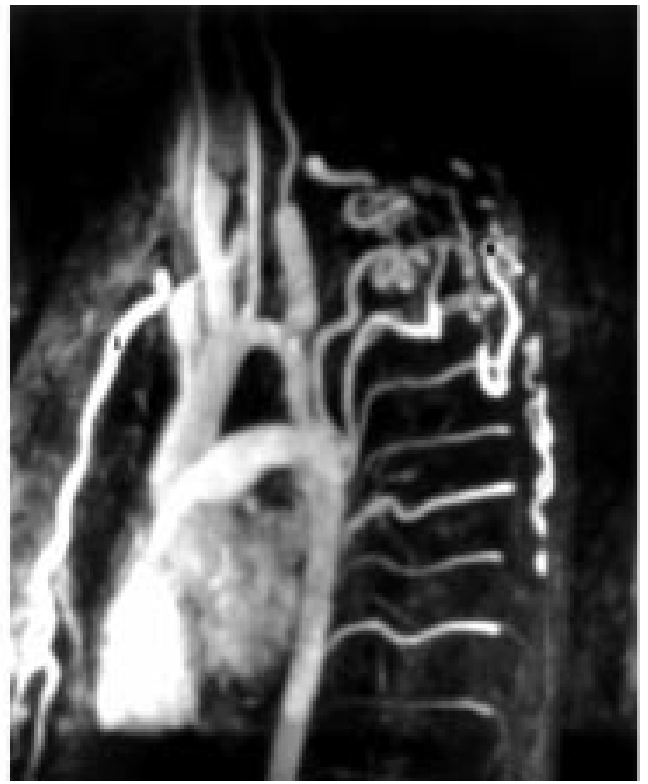

region (right) (i). The parascapular collateralisation ( $\mathrm{s}$ ) and intercostal artery hypertrophy is also clearly visible. Contrast enhanced magnetic resonance angiography is a minimally invasive technique for evaluating suspected coarctation of the aorta providing superior detail to conventional magnetic resonance imaging.
A R O'CONNOR

A R MOODY

C N LUDMAN 